\title{
Nutech Functional Score: A New Functional Scoring System for Patients With Cerebrovascular Accidents
}

\author{
Geeta Shroff
}

\begin{abstract}
Background: Stroke or cerebrovascular accident happens when a part of the brain gets poor supply of blood that leads to either hemorrhage or blockage due to deoxygenation. To measure stroke outcomes, various stroke scales are invented in the last few decades. The present study has compared a novel stroke scale, Nutech functional score (NFS) to that with the most widely used and globally recognized European stroke scale (ESS).
\end{abstract}

Methods: NFS has been designed with 22 different parameters taking into consideration its ability to measure neurological as well as functional impairments associated with stroke. Each symptom has been assigned scores $(1,2,3,4,5)$ that runs in direction from extreme bad (1) to normal (5). The scores are converted to numeric values using an empirical formula. We have compared both the NFS and ESS scores for measuring stroke outcomes.

Results: NFS is able to assess motor, sensory and autonomic parameters for stroke patients. It assesses not only the clinical symptoms but the overall change in quality of life. ESS fails to measure all kinds of functional parameters and it involves tedious calculations.

Conclusion: NFS has proven to be a much simpler and efficient scoring system in comparison with ESS which is a 14-point stroke scale. NFS is a multiple use scale which can measure more parameters than ESS and can be used universally to assess the patients suffering with stroke.

Keywords: Nutech functional score; European stroke scale; Stroke; Cerebrovascular accidents; Human embryonic stem cells

\section{Introduction}

Cerebrovascular accident (CVA) or stroke is a neurological symptom caused by cerebral ischemia or hemorrhage, which if

Manuscript accepted for publication September 22, 2015

Nutech Mediworld, H-8, Green Park Extension, New Delhi 110016, India. Email: geetashroff@hotmail.com

doi: http://dx.doi.org/10.14740/jnr352w not treated or if too severe may lead to death [1]. As per WHO, stroke is defined as "rapidly developing clinical signs of focal (or global) disturbance of cerebral function, with symptoms lasting $24 \mathrm{~h}$ or longer or leading to death, with no apparent cause other than of vascular origin" $[2,3]$.

According to Center of Disease Control (CDC), stroke is the fifth leading cause of death in the United States. In United States alone, every year about 610,000 people are affected by a stroke and 130,000 die each year. The etiology of stroke varies with age, gender, race and ethnicity $[4,5]$.

Based on pathological findings, strokes are of two types: ischemic (due to cerebral infarction) and hemorrhagic (due to disturbances of the cerebral blood circulation). The most common symptom of stroke is sudden weakness or numbness of the face and limbs, most often on one side of the body. Other symptoms include: confusion, difficulty in speaking, walking, writing, visual disturbances and loss of consciousness. The effects of a stroke depend on which part of the brain is injured and how severely it is affected. A very severe stroke can cause sudden death $[2,3]$.

For appropriate patient care and treatment management, it is important to make accurate prediction of stroke outcomes which can assess both the neurological impairment as well as functional health. In the last few decades, various scoring scales have been developed which help in diagnosis and prognosis of stroke. Disability, handicap and improvement in quality of life are the major concerns which are considered to make an efficient scoring scale $[6,7]$. Most of the stroke scoring instruments are usually based on the basis of clinical history of patients such as loss of consciousness, convulsive fits and neurological signs, viz. facial palsy, limb weakness, speech disturbance or visual field defect [8]. Various stroke scales have been developed so far: pre-hospital assessment scales (Cincinnati stroke scale, Los Angeles pre-hospital stroke scale (LAPSS), and ABCD score), acute assessment scales (Canadian neurological scale (CNS), European stroke scale (ESS), Glasgow coma scale (GCS), hemispheric stroke scale (HSS), Hunt and Hess scale, Mathew stroke scale, National Institutes of Health stroke scale (NIHSS), Orgogozo stroke scale (OSS) and Scandinavian stroke scale (SSS)), functional assessment scales (Berg balance, Lawton IADL scale, Rankin scale, and stroke specific quality of life measure (SS-QOL)) and outcome assessment scales (Barthel index, functional independence measurement $\left(\mathrm{FIM}^{\mathrm{TM}}\right)$, and Glasgow outcome 
Table 1. Conversion Table From Categorical Scores to Numeric Range for NFS

\begin{tabular}{lllllll}
\hline \multirow{2}{*}{ No. of scores } & Numeric & \multicolumn{7}{c}{ Categorical scores } \\
\cline { 3 - 7 } & & $\mathbf{1}$ & $\mathbf{2}$ & $\mathbf{3}$ & $\mathbf{4}$ & $\mathbf{5}$ \\
\hline 5 & Score & 0.122 & 0.310 & 0.500 & 0.690 & 0.89 \\
& Range & $0-0.241$ & $0.241-0.379$ & $0.379-0.621$ & $0.621-0.759$ & $0.759-1.00$ \\
3 & Score & 0.167 & 0.500 & 0.833 & - & - \\
& Range & $0-0.333$ & $0.333-0.667$ & $0.667-1.00$ & - & - \\
\hline
\end{tabular}

scale $(\mathrm{GOS}))[6,9-13]$.

Though all these scales are well established and are used globally, they have their own limitations in terms of validity, reliability and sensitivity. Most of these scales have tedious calculations based on the clinimetric principles of interrater reliability, internal consistency and time needed to perform it $[6,14]$.

ESS, a 14-point scaling system is the most popular scale used nowadays to measure stroke. It has major drawback of measuring only limited sensory functions level of consciousness, vision and few selected motor functions like gait and upper and lower body part of body movements. The results interpretation method by ESS is also confined in range of 0 100 , where 0 is the worst condition and 100 is the maximum improvement attained. Thus, ESS can depict results for extreme conditions only; it fails to measure mid-level improvement such as mild, moderate and severe conditions [13]. We have previously reported in our studies, where patients with CVA had shown improvement in using hESC therapy scored with ESS [15].

In this paper, we have described a new functional scoring system, Nutech functional score (NFS) to assess stroke outcomes. It is a 22-point scaling system which not only assesses all the possible known symptoms of stroke but also at every level of improvement.

\section{Material and Methods}

\section{Ethical statement}

Our work has been approved by an Independent Ethics Committee (IEC). The ethics committee approved this procedure for obtaining the consent. The study was conducted in compliance with the "Declaration of Helsinki" in a good clinical practices (GCP). The institutional committee for stem cell research and therapy of Nutech Mediworld, New Delhi, India reported the clinical study to National Apex Committee for Stem Cell Research and Therapy (NAC-SCRT). A verbal, written and video consent was provided by the patients.

\section{Patient study}

The patients diagnosed with stroke were treated in our institute. All possible stroke symptoms were recorded in the history at the time of admission, and were observed and recorded throughout the treatment so as to measure improvement at every level to maintain accuracy and precision. The patients were diagnosed with both NFS and ESS to make the comparison.

The NFS scale scoring system for each parameter ranges between 1 and 5 . A change towards higher score indicates improvement. We have converted the scores into numeric values required for probability studies. The parameters are continuously revised as per the new symptoms observed in patients.

\section{Results}

NFS is a numeric scoring scale and can measures score for 22 different symptoms/parameters of stroke. Each parameter has been assigned a score ranging from 1 to 5 . The score " 1 " stands for extreme gravity of the condition and score " 5 " is assigned for normal. The slightest improvement in any symptom can be easily measured at different sublevels of 2, 3 and 4 .

It is a very simple method where all the known parameters, functional as well as neurological have been selected. Not afflicted (NAA) and not existing (NE) conditions are also included.

Supplementary 1 (www.neurores.org) lists the NFS parameters and the scores assigned as per the severity of condition.

This scoring system lies in a hypothetical categorical range of 0.5 and 5.5, so as to keep the scores equidistant from each other and continuous. In probability-based studies, a range of $(-1,1)$ or at least the range of $(0,1)$ is required, so the scores are converted to respective numeric values. The " 0.5 " and " 5.5 " of hypothetical categorical range $(0.5,5.5)$ is considered as " 0 " and " 1 " of the $(0,1)$ numeric scale, respectively.

The configuration can be used for one symptom and has also been used for our patients with cerebral palsy [16].

For $Y_{c}=$ categorical score and $Y_{n}=$ numeric score, the relationship at any value of " $\mathrm{x}$ " is expected as: $Y_{c}+0.5 / 0.5=$ $\mathrm{x}=\mathrm{Y}_{\mathrm{n}}+0.166 / 0.96$.

The conversion of the scores is presented in Table 1.

This layout can be used universally to convert five categorical scores (range 0.5 - 5.5) to five numeric scores (range $0-1$ ) or three categorical scores to three numeric scores (range $0-1$ ) depending upon the symptoms of parameters assessed by NFS.

\section{Discussion}

Clinically, the stroke patient has various paresis and/or paraly- 
Table 2. Differences Between NFS and ESS

NFS
It includes 22 parameters.
All motor, sensory and autonomic parameters are covered.
Requirement of catheterization is a very important parameter to be
considered at the time of discharge of patients. It is included in NFS.
It includes important sensory parameters
like bowel and bladder sensations.
The scoring is done on 1 - 5 levels. Extreme conditions are given
scores of 1 and 5 . Mild, moderate and severe conditions are given 4 ,
3 and 2 scores respectively.

There are two extreme levels and the number of intermediate levels is mostly three in NFS.

As all the parameters in NFS scoring system have been scored on a scale of $1-5$ in the range of $0.5-5.5$, which makes NFS an ordinal, it provides complete information regarding the condition of the patient before and after the therapy.

It is important to note that NFS does not include " 0 " as a score. Therefore analytical work based on "count" stays unaffected.

The parameters are more elaborative in NFS, where the parameters are further divided separately with co-existing conditions, e.g. for spasticity which is a feature of altered skeletal muscle performance with a combination of paralysis, increased tendon reflex activity and hypertonia are explained in NFS. This parameter has been divided into three separate parameters for associated conditions of muscle deformity, tonicity and with clonus.

\section{ESS}

It includes 14 parameters.

Mostly motor and very few sensory parameters are covered. Autonomic parameters like writing, breathing and swallowing are missing.

Requirement of catheterization parameter is missing in ESS.

Sensory parameters like bowel and bladder sensations are missing in ESS.

Though in ESS, there is a broader classification of scoring; the scoring system is not uniform, as all the parameters are not assigned the similar pattern of scores/points for extreme as well as intermediate levels. In few parameters of ESS scale, the maximum score is either assigned as 4, 8 or 10. Like for level of consciousness and gait, the maximum score has been assigned 10, though for extension of wrist, dorsiflexion of foot, the maximum score is 8 . Similarly, for leg flexion and positioning of arms, the highest score is 4 .

There is non-uniformity in number of intermediate levels like, for vision there are just two extreme levels, for fingers and facial movements, there is just one sublevel and two extreme levels, for speech and gaze there are four levels and for gait and speech, there are five sublevels.

Final data are computed within the range of 0 and 100 which depict minimum to maximum level of improvement. Mid level of improvement remains undisclosed.

ESS does not have such elaborative classification for a single parameter. sis of the body. Apart from the various nuclear imaging tests recommended for brain such as magnetic resonance imaging (MRI), computerized axial tomography (CT) scan and single photon emission computed tomography (SPECT) imaging just after the admission of patient and also during the treatment, the patient is needed to be evaluated for the assessment of other functional symptoms like vision, movement, sensation and speech for further patient care and treatment management $[17,18]$. This is done by various scales that are internationally recognized and are based on the principle of clinimetrics and explain the measures of validity, reliability, and responsiveness. These scales help in assessing the patient's condition but they only explain the motor health of the patient $[6,14]$.

The use of these scales is limited because the calculations are complicated and not indicative of the actual extent of the affectation [19]. Like in ESS, the prime focus is on measurement of motor functions such as body movement and very few sensory parameters like speech, vision and level of con- sciousness are included. However, NFS scale is much simpler to use and has lesser calculations and the scores can be easily subtracted or added. The scale has been designed keeping in consideration the important parameters which can measure the functional as well as neurological complications of the patients undergoing treatment for stroke. ESS.

Table 2 lists some important differences between NFS and

In comparison to ESS, the parameters in NFS are more defined. It is able to measure the functional independence of patients throughout the treatment, i.e., starting at admission, during rehabilitation, and upon discharge. The motor functions such as: standing and sitting balance, facial palsy, flaccidity, atrophy and spasticity; sensory functions like bowel, bladder sensations, requirement of catheterization and autonomic functions like breathing, swallowing and writing can be measured easily. Each parameter is defined into sublevels and some parameters are further classified with the co-existing 
Table 3. Comparison of NFS and ESS Scores Before and After Treatment

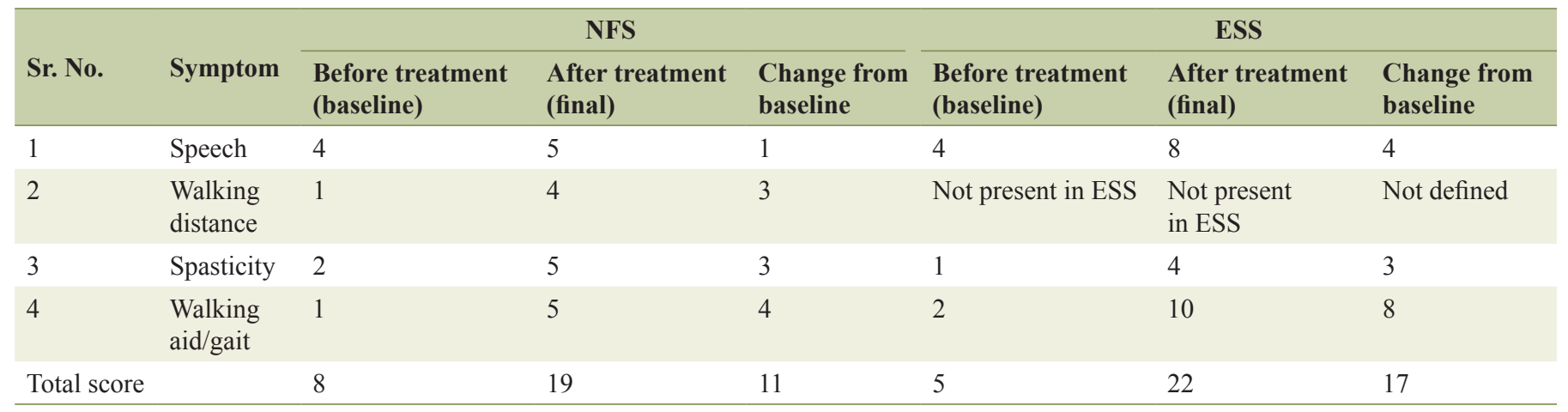

NFS: speech: 4 - slurred but comprehensive, 5 - normal speech; walking distance: 1 - cannot walk, 4 - can walk up to 100 m; spasticity: 2 - considerable increase in the muscle tone, passive movement difficult, 5 - less spasticity or reached normalcy; walking aid/gait: 1 - walker with elbow support, 5 - no aid required.

ESS: speech: 4 - severe word finding difficulties, conversation is difficult, 8 - normal speech; spasticity: 1 - legs and arms drifts to bed within $5 \mathrm{~s}$ but not immediately, 4 - legs and arms maintain position for $5 \mathrm{~s}$; walking aid/gait: 2 - cannot walk but can stand supported, 10 - normal.

conditions, like, for spasticity which is a feature of altered skeletal muscle performance with a combination of paralysis, increased tendon reflex activity and hypertonia are explained in NFS. This parameter has been divided into three separate parameters for associated conditions of muscle deformity, tonicity and with clonus. Also, each parameter has been further classified in mild, moderate and severe levels of impairment. This makes the assessment of signs and symptoms of CVA easy and minor improvements or deteriorations can be tapped. Unlike NFS, ESS does not specify if motor or sensory function is non-contiguous or on one side of the body. ESS is unable to depict the gross condition of the patients, it fails to determine if patients require calipers to support while walking and other important parameters like difficulty in breathing, bowel and bladder sensation and requirement of catheterization which are useful to predict the appropriate time of discharge of patients, are missing in ESS scoring system $[13,20]$. The scoring system of NFS is also very easy and less tedious than ESS. In NFS, the extreme levels are given scores 1 and 5, the sublevels mild, moderate and severe have been given scores 4,3 and 2 respectively. Though in ESS, there is a broader classification of scoring, the scoring system is not uniform, as all the parameters are not assigned the similar pattern of scores/points for extreme as well as intermediate levels. In few parameters of ESS, the maximum score is either assigned as 4,8 or 10 . Like for level of consciousness and gait, the maximum score has been assigned 10, though for extension of wrist, dorsiflexion of foot, the maximum score is 8 . Similarly, for leg flexion and positioning of arms, the highest score is 4 .

The variability lies not only in maximum score but also in number of intermediate levels like, for vision there are just two extreme levels, for fingers and facial movements, there is just one sublevel and two extreme levels, for speech and gaze there are four levels and for gait and speech, the levels are five. Though, the interpretation of results is done similarly as that of NFS by summing up the points attained at the end for all the 14 parameters but the final data are computed within the range of 0 and 100 which depicts minimum to maximum level of improvement [13].
As all the parameters in NFS scoring system have been scored on a scale of 1 to 5 in the range of 0.5 to 5.5 , which makes NFS an ordinal, it provides complete information regarding the condition of the patient before and after the therapy. It is important to note that NFS does not include "0" as a score. Therefore analytical work based on "count" stays unaffected.

To understand more on NFS, let us take an example of a patient with CVA. The NFS scores were noted at the time of admission (baseline) and compared with the final scores, i.e. after completing the treatment. The same patient was simultaneously analyzed on ESS for baseline as well as final scores. The baseline scores from both the scales were compared with the final scores at the end.

A 57-year-old male patient was admitted to our facility in December 2004, under the subconscious condition of CVA with left hemiparesis. The patient had a previous history of cerebral infarction in the left side of brain and had long term hypertension. At the time of admission, the immediate symptoms noted were difficulty in walking, poor standing balance and muscle atrophy and spasticity in left upper and lower limbs. The previous CT scans and MRI reports showed a loss of gray-white differentiation in superficial cortical infarction. The patient was on conventional treatment of anti-platelet aggregating agents and anti-coagulants.

To make a comparison between NFS and ESS, we selected few parameters which were common in both NFS and ESS. We measured the baseline scores of the patient before starting the treatment by both the scales for following parameters: speech, gait, walking distance and spasticity. The scores were recorded during the regular intervals of the treatment to check the change in improvement and after the completion of first phase of treatment. The change in baseline scores from final scores was also recorded. Both the scales had shown improvement with the treatment given, as there was an increase in scores compared to the baseline.

Table 3 shows the parameters studied before and after the treatment by both NFS and ESS.

Besides the above comparison, various other functional parameters like writing, sensory, bowel and bladder movement 


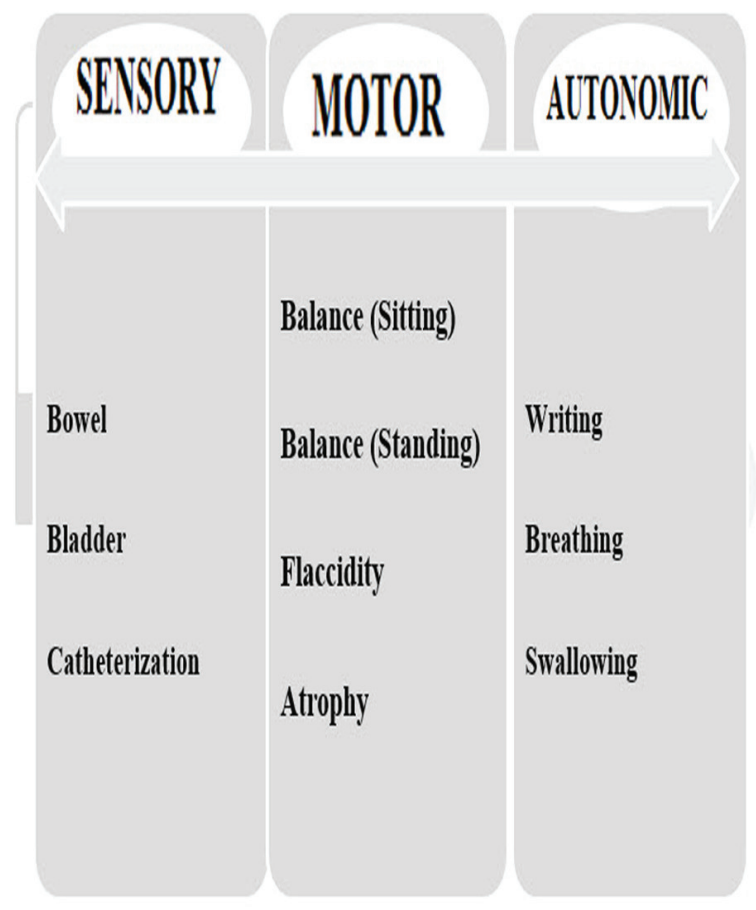

Figure 1. Parameters are assessed in NFS but not in ESS.

which ESS failed to measure were also scored on NFS scale. ESS.

Figure 1 shows the comparison of NFS parameters with

Though unique, easy to use and better at scoring the patients with CVA, NFS is used at our facility only; its novel methodology and use still need to be evaluated by other healthcare professionals in India and worldwide. As the scoring system is based on conversion in numeric values which are universally acceptable as they are pure numbers staying within the range of $(0,1)$, thus are compatible to all variables that distribute likewise including the probability distributions. Thus, NFS can be considered as a unique tool to assess the improvement in patients with CVA.

\section{Acknowledgement}

The authors acknowledge Dr. J. K. Barthakur for statistical support, all the doctors, staff and patients of the Nutech Mediworld. The authors also acknowledge Knowledge Isotopes Pvt. Ltd. (http://www.knowledgeisotopes.com) for the writing support.

\section{Conflict of Interest}

The authors have no conflict of interest.

\section{References}

1. GoodDC.CerebrovascularDisease: http://www.ncbi.nlm.
nih.gov/books/NBK378/pdf/Bookshelf_NBK378.pdf. Last accessed on 8 June 2015.

2. Stroke, Cerebrovascular accident. Available at: http:// www.who.int/topics/cerebrovascular_accident/en/ , last accessed on 26 JUN 2015.

3. Thomas Truelsen SB, Colin Mathers. The global burden of cerebrovascular disease . Available at:http://www.who. int/healthinfo/statistics/bod cerebrovasculardiseasestroke.pdf. Last accesed on- $2 \overline{6}$ JUN 15. 2000.

4. Stroke Facts. Available at: http://www.cdc.gov/stroke/ facts.htm Last Accessed on 26 JUN 15.

5. Know the Facts about Stroke. Available at- http://www. cdc.gov/stroke/docs/consumered_stroke.pdf. Last accessed on- 26 JUN 16.

6. De Haan R, Horn J, Limburg M, Van Der Meulen J, Bossuyt P. A comparison of five stroke scales with measures of disability, handicap, and quality of life. Stroke. 1993;24(8):1178-1181.

7. Ayis SA, Coker B, Rudd AG, Dennis MS, Wolfe CD. Predicting independent survival after stroke: a European study for the development and validation of standardised stroke scales and prediction models of outcome. J Neurol Neurosurg Psychiatry. 2013;84(3):288-296.

8. Nor AM, Davis J, Sen B, Shipsey D, Louw SJ, Dyker AG, Davis M, et al. The Recognition of Stroke in the Emergency Room (ROSIER) scale: development and validation of a stroke recognition instrument. Lancet Neurol. 2005;4(11):727-734.

9. Stroke Assessment Scales. Available at- http://www. strokecenter.org/professionals/stroke-diagnosis/strokeassessment-scales/. Last accessed on 26 JUN 15.

10. Adams HP, Jr., Davis PH, Leira EC, Chang KC, Bendixen BH, Clarke WR, Woolson RF, et al. Baseline NIH Stroke Scale score strongly predicts outcome after stroke: A report of the Trial of Org 10172 in Acute Stroke Treatment (TOAST). Neurology. 1999;53(1):126-131.

11. Blum L, Korner-Bitensky N. Usefulness of the Berg Balance Scale in stroke rehabilitation: a systematic review. Phys Ther. 2008;88(5):559-566.

12. Cote R, Hachinski VC, Shurvell BL, Norris JW, Wolfson C. The Canadian Neurological Scale: a preliminary study in acute stroke. Stroke. 1986;17(4):731-737.

13. Hantson L, De Weerdt W, De Keyser J, Diener HC, Franke C, Palm R, Van Orshoven M, et al. The European Stroke Scale. Stroke. 1994;25(11):2215-2219.

14. Harrison JK, McArthur KS, Quinn TJ. Assessment scales in stroke: clinimetric and clinical considerations. Clin Interv Aging. 2013;8:201-211.

15. Shroff G BJ. Safety and Efficacy of Human Embryonic Stem Cells for the Treatment of Cerebrovascular Accident: A Case Series. In Press Global Journal of Medical Research 2015.

16. Geeta Shroff JKB. Nutech Functiona Score (NFS), A new scoring system to assess the level of impairment in patients with cerebral palsy. International Archives of Medicine. 2015;9.

17. Spilker J, Kongable G, Barch C, Braimah J, Brattina P, Daley S, Donnarumma R, et al. Using the NIH Stroke Scale to assess stroke patients. The NINDS rt-PA Stroke 
Study Group. J Neurosci Nurs. 1997;29(6):384-392.

18. Merali Z, Wong T, Leung J, Gao MM, Mikulis D, Kassner A. Dynamic contrast-enhanced MRI and CT provide comparable measurement of blood-brain barrier permeability in a rodent stroke model. Magn Reson Imaging. 2015;33(8):1007-1012.

19. Sanders S, Flaws D, Than M, Pickering JW, Doust J, Glasziou P. Simplification of a scoring system maintained overall accuracy but decreased the proportion classified as low risk. J Clin Epidemiol. 2015.

20. Joseph A. Cloud DCJ, Tricia A. Lauinger. Functional Independence Measurement Scale: Analysis of variables to determine predictability to stroke patient's discharge site. Available at http://scholarworks.gvsu.edu/cgi/viewcontent.cgi? article $=1214 \&$ context $=$ theses. Last accessed on30 JUN 15 